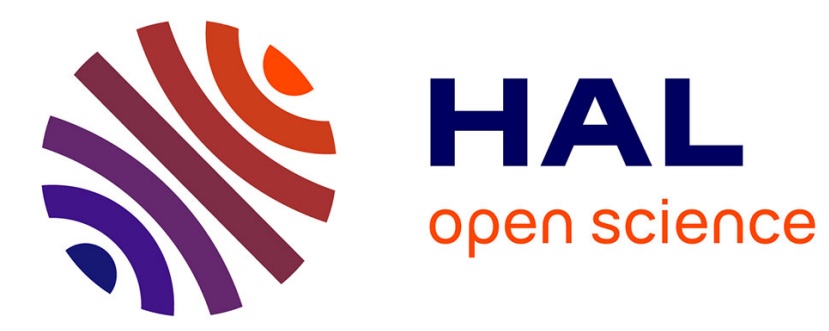

\title{
Microenvironment signaling driving lymphomagenesis
}

Léa Verdière, Frédéric Mourcin, Karin Tarte

\section{To cite this version:}

Léa Verdière, Frédéric Mourcin, Karin Tarte. Microenvironment signaling driving lymphomagenesis. Current Opinion in Hematology, 2018, 25 (4), pp.335 - 345. 10.1097/MOH.0000000000000440 . hal01860228

\section{HAL Id: hal-01860228 \\ https://hal.science/hal-01860228}

Submitted on 20 Sep 2018

HAL is a multi-disciplinary open access archive for the deposit and dissemination of scientific research documents, whether they are published or not. The documents may come from teaching and research institutions in France or abroad, or from public or private research centers.
L'archive ouverte pluridisciplinaire HAL, est destinée au dépôt et à la diffusion de documents scientifiques de niveau recherche, publiés ou non, émanant des établissements d'enseignement et de recherche français ou étrangers, des laboratoires publics ou privés. 


\section{Microenvironment signaling driving lymphomagenesis}

Lea Verdière $^{1}$, Frederic Mourcin ${ }^{1}$, and Karin Tarte Te $^{1,2}$

${ }^{1}$ UMR_S 1236, Université Rennes 1, INSERM, Etablissement Français du Sang, Labex IGO, F-35000, Rennes, France

${ }^{2}$ SITI, Pôle de Biologie, CHU Pontchaillou, F-35000, Rennes, France

Correspondence: Karin TARTE

INSERM UMR U917

Faculté de médecine

2 avenue du Pr Léon Bernard

35043 RENNES, FRANCE

karin.tarte@univ-rennes1.fr 


\section{Abstract}

\section{Purpose of review}

Besides the recent progresses in the description of the genetic landscape of B-cell non-Hodgkin lymphomas, tumor microenvironment has progressively emerged as a central determinant of early lymphomagenesis, subclonal evolution, drug resistance, and late progression/transformation. The purpose of this review is to outline the most recent findings regarding malignant $\mathrm{B}$-cell niche composition and organization supporting direct and indirect tumor-promoting functions of lymphoma microenvironment.

\section{Recent findings}

Lymphoma supportive niche integrates a dynamic and orchestrated network of immune and stromal cell subsets producing, with a high level of spatial and kinetic heterogeneity, extracellular and membrane factors regulating tumor migration, survival, proliferation, immune escape, as well as microarchitecture and mechanical constraints. Some recent insights have improved our understanding of these various components of lymphoma microenvironment, taking into account the mechanisms underlying the coevolution of malignant and non-malignant cells within the tumor niche.

\section{Summary}

Deciphering tumor niche characteristics, functions, and origin could offer new therapeutic opportunities through the targeting of pivotal cellular and molecular components of the supportive microenvironment, favoring immune cell reactivation and infiltration, and/or limiting tumor retention within this protective niche.

Keywords: tumor niche, immune escape, BCR, extracellular matrix, cancerassociated fibroblasts 


\section{INTRODUCTION}

B-cell Non-Hodgkin's Lymphomas (B-NHL) are a group of heterogeneous neoplasias corresponding to clonal expansion of mature $B$ cells arrested at various stages of differentiation and disseminated preferentially within secondary lymphoid organs and bone marrow (BM) [1]. The current World Health Organization identifies more than 30 entities with specific phenotypic, genetic, and clinical features as well as variable degrees of dependence on external stimuli for survival and proliferation. Follicular lymphoma ( $F L$ ) and Diffuse large B-cell lymphomas (DLBCL), the two most common B-NHL, are both derived from germinal center (GC) B cells but are characterized by opposite patterns of tumor niche composition and organization, making them interesting models to understand how these two parameters could influence lymphomagenesis dynamic $[2,3]$.

$\mathrm{FL}$ is the paradigm of a B-cell malignancy dependent on a GC-like permissive microenvironment including $\mathrm{CD} 4^{\text {pos }} \mathrm{T}$ cells, myeloid cells, and stromal cells with specific phenotypic and functional features that could be found admixed with malignant $B$ cells within invaded lymph nodes ( $L N)$ and BM. In agreement, numerous predictive biomarkers in FL reflect tumor niche composition and organization rather than genetic features of tumor cells. Moreover, genetic hits in FL are not only related to $B$ cell-intrinsic effects but could support interactions with surrounding non-B cells. Conversely, DLBCL have been proposed as less dependent on LN architecture and the most popular prognostic classification of DLBCL tumors, i.e. GC B cell (GCB)versus activated $B$ cell $(A B C)-D L B C L$, relies on tumor cell genetic characteristics reminiscent of their putative cell of origin and molecular transformation pathways. Importantly, both FL and DLBCL tumors are under a constant selective pressure from the immune system as revealed by the numerous genetic hits favoring immune escape.

Other reviews provide a description of FL/DLBCL genomic landscape and clonal architecture and detail the general concept of pro- versus anti-tumoral microenvironment $[4,5]$. This review will focus on the more recent concepts of tumor niche-derived cellular and non-cellular supportive signals in B-cell lymphomas, how they are triggered, and their potential clinical impact on patient stratification and treatment. 


\section{IMMUNE ESCAPE MECHANISMS UNDER THE SPOTLIGHTS}

In B-cell lymphomas, malignant cells are MHC class II-expressing professional antigen-presenting cells and induction of anti-tumor innate and adaptive immunity has been well documented. In agreement, FL has been one of the first tumor subtype in which tumor vaccines have been tested with a good biological response [6] and both tumor-associated macrophages (TAM) and NK cells largely contribute to the clinical efficacy of anti-CD20 antibodies. However, several immune escape mechanisms have been uncovered including: i) reduction of tumor immunogenicity; ii) inhibition of immune effectors; and iii) infiltration by immunosuppressive cells (Figure 1).

First, tumor cells decrease their expression of MHC molecules to avoid recognition by CD8 and CD4 T cells. B2M genetic inactivation resulting in loss of MHC class I is more frequent in transformed FL and DLBCL whereas lack of MHC class II expression is a common feature across lymphoma subtypes [7-9]. Interestingly early loss-of-function mutations of CREBBP found in about $60 \%$ of $\mathrm{FL}$ and $15 \%$ of DLBCL have been recently proposed as the major mechanism supporting the downregulation of MHC class II and the corresponding decreased T-cell activation and in situ infiltration [7]. Mechanistically, CREBBP mutations disable H3K27 acetylation and favor BCL6-dependant transcriptional repression of genes regulating MHC class II expression and antigen presentation [10].

The second driving mechanism of immune escape is the reduced anti-tumoral T/NK cell activity. Both FL- and DLBCL-infiltrating cytotoxic T cells display an upregulation of coinhibitory receptors, an impaired LFA-1-dependent motility, and a decreased capacity to form functional immunological synapses with malignant cells [11-17]. Importantly, LAG-3, TIM-3, and TIGIT have emerged recently as good markers of Tcell exhaustion in B-cell lymphoma whereas PD-1 expression is not sufficient to distinguish exhausted from activated T cells, in particular in FL [18]. An important question remains the nature of the ligand-expressing cells. In fact, only $20-30 \%$ of DLBCL B cells, essentially some ABC-DLBCL and EBV ${ }^{\text {pos }}$ DLBCL, and no FL B cell express PD-L1 whereas infiltrating PD-L1 ${ }^{\text {pos }}$ myeloid cells could be detected in both DLBCL and FL [19]. The TIGIT ligands CD122 and CD155 were detected on follicular dendritic cells and endothelial cells inside FL follicles [13]. Of note, another important parameter to take into account when considering anti-tumor T-cell response is the impact of TCR repertoire. Intratumoral TCR repertoire was reported to be abnormally 
narrow in DLBCL and this restriction of TCR diversity was associated with adverse outcome [20]. Finally, NK cells could also be inhibited within lymphoma cell niches, either through expression of PD-1 or through interaction with lectin-like transcript 1 (LLT1), the ligand for the NK inhibitory receptor CD161 recently found to be strongly expressed by FL and GC-DLBCL malignant B cells [21].

FL/DLBCL are also characterized by an expansion of immunosuppressive cells including Treg and myeloid-derived suppressor cells (MDSC). CD25 $5^{\text {hi }}$ Treg subset is strongly enriched in FL compared to reactive lymphadenitis and DLBCL and specifically includes classical CXCR5 ${ }^{\text {neg }} P D-1^{\text {neg }}$ Treg but also a huge proportion of $\mathrm{CXCR}^{\mathrm{pos}} \mathrm{ICOS}^{\mathrm{pos}} \mathrm{PD}-1^{\text {pos }}$ follicular regulatory $\mathrm{T}$ cells $(\mathrm{Tfr})[22,23]$. Tfr have been previously identified within normal GC and interfere with T-cell help produced by follicular helper T cells (Tfh) thus negatively controlling the GC reaction [24]. In FL, Treg/Tfr are able to suppress not only $C D 4^{\text {pos }} \mathrm{T}$ cells, including Tfh, but also FL B cells, suggesting that they could have indirect and direct anti-tumor activity [23]. Conversely, they could also inhibit cytotoxic $T$ cells that are essentially found at the GC border in FL in agreement with a strongly suppressive microenvironment within invaded follicles $[25,26]$. Such opposite functions could explain controversial results concerning the prognostic impact of FOXP3 ${ }^{\text {pos }}$ Treg number and localization. This skewing of T-cell response towards regulatory phenotype is at least partly orchestrated by malignant FL B cells through the production of high amounts of the Treg recruiting chemokine CCL22, and the expression of CD70 and ICOS-L $[23,27,28]$. Besides Treg, suppressive myeloid cells have emerged as important drivers of immune escape in B-cell lymphomas, as highlighted by the negative prognostic impact of i) a high number of circulating myeloid cells, including monocytes and neutrophils; ii) a high number of $C D 68^{\text {pos }}$ or $C D 163^{\text {pos }}$ myeloid cells within lymphoma niches; iii) a high level of markers related to myeloid suppressive functions, including soluble PD-L1, IL-10, and indoleamine-2,3 dioxygenase [29-31]. Recently, specific attention has been paid to the role of MDSC in B-cell lymphomas. Two main cell subsets, monocytic (M)-MDSC and granulocytic (G)-MDSC, have been demonstrated to play potent immunosuppressive functions in cancers and inflammatory diseases. Both circulating M-MDSC and G-MDSC were found elevated in lymphoma patient peripheral blood and their abundance has been variably proposed as predictive of clinical outcome [32,33]. The function of M-MDSC has been particularly well studied in DLBCL and was ascribed to their expression of 
S100A12, IL-10, and PD-L1, unlike to arginase 1 and IDO immunosuppressive enzymes [32]. It has been demonstrated in murine models that MDSC could differentiate into TAM at the tumor site but the precise relationship between MDSC and TAM in human lymphomas remain unclear. Lymphoma-derived TAM have been shown to produce IDO and interleukin-4-induced gene 1 (IL4I1) enzymes associated with tryptophan and phenylalanine catabolism, respectively [31,34]. Finally it is important to keep in mind that cancer-associated fibroblasts (CAF) could contribute to immune escape as recently highlighted in solid cancers [35]. Interestingly, FLinfiltrating stromal cells overexpress PGE2, a well-known immunosuppressive factor [36], and stromal cells were proposed to favor B-cell lymphoma growth in a murine model of lacrimal gland lymphoma through the recruitment of Treg and MDSC [37].

\section{BCR AND MICROENVIRONMENT: A REVISITED PARAdIGM}

The central role of $B C R$ signaling in lymphomagenesis is now well documented. $A B C-D L B C L$ tumors, the most aggressive forms of DLBCL, are addicted to NF- $\kappa B$ activation and mutations in BCR components or downstream signaling effectors leading to a chronic active BCR signaling have been involved in this oncogenic NF$\kappa B$ activity [38]. Interestingly, chronic active $B C R$ signaling retains the hallmarks of normal antigen-dependent signaling and a role for self-antigen recognition in the initiation and maintenance of $B C R$ activation in $A B C-D L B C L$ has been recently proposed [39]. In agreement, some of the recurrent genetic hits in ABC-DLBCL, such as the gain-of-function mutations in $C D 79 A / B$, cannot trigger $B C R$ signaling the novo but amplify it. By contrast, GC-DLBCL are dependent on activation of PI3K/Akt by antigen-independent tonic BCR signaling, a process that could be favored by PTEN inactivation [40]. Of note whereas GC-DLBCL harbor an IgG BCR, ABC-DLBCL retain expression of an $\operatorname{IgM~BCR}$ as a consequence of deletions in the $\mathrm{S} \mu$ region of the productive IGH allele [41]. The unique cytoplasmic tails of IgM and IgG BCR produce qualitatively different signaling outputs in normal antigen-experienced $B$ cells [42]. Moreover, switched memory $B$ cells are prone to generate a large number of plasma cells whereas $\operatorname{lgM}^{\text {pos }}$ memory $B$ cells reinitiate a $G C$ reaction upon antigenic challenge $[43,44]$. It would be important to understand whether differences between IgM versus IgG signaling contribute to differences between GC- and ABC-DLBCL pathogenesis. Interestingly, a role for tumor microenvironment has recently emerged 
in the activation of BCR in FL patients. Specifically, although less than $25 \%$ of $\mathrm{FL}$ $B C R$, essentially of IgG isotype, are supposed to be self-reactive [45], FL BCR is characterized by i) a selective pressure to retain an IgM BCR despite active class switch recombination process; ii) the introduction of $\mathrm{N}$-glycosylation sites within variable regions of immunoglobulin genes during somatic hypermutation in more than $80 \%$ of FL cases [46]. The maintenance of IgM BCR could contribute to the frozen GC phenotype of FL B cells that is established very early during lymphomagenesis process and is associated with a high risk of additional genetic events [47][48]. Moreover, IgM FL BCR crosslinking triggers a stronger BCR activation than IgG FL BCR [49]. Importantly, these $\mathrm{N}$-glycosylation sites are occupied by unusual glycans terminating at high mannose allowing an interaction with DC-SIGN on the surface of FL TAM and a delayed but long-lasting BCR aggregation and activation in the absence of surface immunoglobulin endocytosis [49,50]. Of note bacteria-derived lectins have also been shown to trigger FL BCR aggregation in vitro [51]. In this context, recurrent genetic alterations are thus associated with an increased capacity of tumor cells to interact with tumor microenvironment. Mutations in BCR/NF-KB signaling pathways have been recently described in about 30\% of $\mathrm{FL}$ patients but their biological and clinical significance remain to be elucidated [52].

\section{Tumor Permissive Cell Microenvironment: A Key role for CAF}

Apart from BCR signaling, lymphoma microenvironment also delivers a combination of survival and growth signals that promote lymphoma development and drug resistance (Figure 1). It has long been assumed that tumor-supportive $\mathrm{CD} 4^{\mathrm{pos}} \mathrm{T}$ cells are specifically amplified in FL, unlike DLBCL. FL-infiltrating $C D 4^{\text {pos }} \mathrm{T}$ cells display a $\mathrm{CD} 10^{\text {pos }}$ Tfh phenotype, and overexpress $\mathrm{CD} 40 \mathrm{~L}$ and IL-4 involved in the direct stimulation of FL B cell survival and stimulating TAM-B cell crosstalk [22,53]. Indeed, Tfh-derived CD40L stimulates the capacity of normal and malignant $B$ cells to respond to macrophage-derived IL-15 [54] whereas IL-4 increases the expression of DC-SIGN on macrophages and membrane IgM on malignant $B$ cells thus favoring BCR/DC-SIGN interaction [49]. More recently, two other cell subsets, potentially involved in both FL and DLBCL, have been considered. The first one is tumorinfiltrating neutrophils (TAN). Whereas TAM have been regularly presented as a major component of lymphoma cell niches, TAN have only been recently studied in these diseases. Interestingly, DLBCL B cells produce IL-8 and recruit neutrophils 
able in turn to protect them from spontaneous and drug-induced apoptosis through expression of a Proliferation-Inducing Ligand (APRIL) and CD11b [55,56]. A high level of IL-8 could also be detected within FL cell niche even if neutrophils do not directly rescue primary FL B cells from apoptosis in vitro [57]. However, TAN could contribute to the activation of stromal cells, inducing their commitment into a FLsupportive phenotype.

In fact, tumor-promoting activities of CAF, including cancer cell proliferation and invasion, inflammation, and drug resistance, and their potential as attractive therapeutic targets have been recently described in solid cancer. FL CAF have focused more attention than their DLBCL counterpart and have been described as lymphoid-like stromal cells exhibiting specific phenotypic and transcriptomic features (Figure 2). In particular, an upregulation of CXCL12 has been recently highlighted in LN and BM FL-CAF contributing to B-cell homing and adhesion to protective cell niches [58]. CAF directly support malignant FL and DLBCL B-cell growth in vitro through a panel of mechanisms only partially solved, including the production of hedgehog ligands and BAFF, and the upregulation of $A B C$ multidrug resistance transporters and C-MYC/HDAC6 loop in tumor cells [59]. Recently an elegant 3D organotypic culture model functionalized with variable integrin specific ligands was used to support a role for the ligand of $\alpha 4 \beta 1$ (VLA-4) integrin, i.e. VCAM-1, a wellknown marker of mature lymphoid stromal cells, in GC B-cell lymphoma growth [60]. Besides this direct B-cell supportive function, FL-CAF were proposed as organizers of FL cell niche and their overexpression of CCL2 and IL-8 trigger the recruitment of monocytes and neutrophils [57,61]. Interestingly, FL-CAF could protect recruited neutrophils from apoptosis and convert recruited monocytes into proangiogenic and anti-inflammatory TAM-like cells. Similarly, FL-CAF were proposed to favor Tfh/Tfr polarization and survival ([62] and unpublished data). New studies based on the analysis of ex-vivo sorted stromal cells will be useful to better understand B-NHL CAF heterogeneity and function.

\section{TUMOR Niche ARchitecture: A role for Extracellular Matrix AND EXTRACELLULAR Vesicles}

Besides adhesive and chemotactic factors expressed by stromal cells, little attention has been given to the acellular components of the reticular fiber networks that form the scaffold of normal and malignant secondary lymphoid organs. In addition to cell 
adhesion molecules, extracellular matrix (ECM)-derived integrin ligands, including $\alpha 3 \beta 1$ ligands, have been proposed to impact normal GC B-cell fate and survival [63] but their role in FL/DLBCL remains to be fully explored. A recent study has uncovered the importance of the matricellular protein secreted protein acidic and rich in cysteine (SPARC) in lymphoma niches. SPARC, a regulator of collagen deposition and organization, was found expressed not only in follicles but also in invaded BM paratrabecular areas of FL and DLBCL and may thus represent a common regulatory element in both BM and LN lymphoma niches [64]. Sparc deletion in mice is associated with defective collagen assembly and a loss of neutrophil inhibitory signal, promoting aberrant crosstalk between neutrophil extracellular traps and B cells and favoring transition from autoimmunity to lymphoma [65]. Overexpression of SPARC was associated with a good prognosis in DLBCL [66] whereas an altered pattern of SPARC expression was found in early FL patients [65]. Considering ECM remodeling components, it has been recently demonstrated that heparanase, the predominant endoglycosidase able to cleave heparan sulfate side chains of proteoglycans, is strongly expressed in about $50 \%$ of $\mathrm{FL}$ and DLBCL [67]. Heparanase triggers ECM disassembly and increases bioavailability of growth factors bound to heparan sulfates. Interestingly, heparanase-neutralizing antibodies inhibit B-cell lymphoma growth and metastasis in mouse xenograft models without direct cytotoxic activity. Finally ECM organization and mechanical forces also regulate 3D tumor architecture and impact tumor evolution. Mechanotransduction may originate from two mechanisms inside tumors: external mechanical forces (i.e. compressive stress) and/or internal mechanical forces (i.e. cell-generated contractility). Whereas recent studies based on multicellular aggregates of lymphoma B cells have proposed to evaluate some of these parameters [68], they did not include stromal cells and compressive forces thus limiting their potential impact. Interestingly, normal LN expansion and contraction specifically rely on the podoplanin-dependent contractile activity of lymphoid stromal cells [69] and podoplanin is downregulated in FL LN [58], in association with a reduced tissue stiffness and expansion of malignant $B$ cells in enlarged LN. Finally, it is important to keep in mind that ECM density and orientation also impact T-cell recruitment within tumor, as initially highlighted in lung cancer [70], a process that could both affect pro- and anti-tumoral $\mathrm{T}$ cells in B-cell lymphomas.

Extracellular vesicles (EV), including microvesicles and exosomes, represent a new component of B-cell/niche crosstalk. Released by tumor cells, they could encapsulate 
different cellular components, including proteins, DNA, or noncoding RNA, and alter the phenotype and functional properties of distant cells. Even if they have been less studied than EV from solid tumors, recent interesting studies proposed that EV produced by malignant $B$ cells could exert different roles including: i) the induction of CAF differentiation, as proposed in chronic lymphocytic leukemia [71]; ii) the protection of tumor B cells from rituximab through the trapping of anti-CD20 antibody [72]; iii) the transfer of the constitutively active membrane-associated protein MyD88 ${ }^{\mathrm{L} 265 \mathrm{P}}$ leading to the propagation of proinflammatory stimuli in recipient surrounding cells [73]. Additional evaluation of the composition and specific roles of lymphoma-derived EV as well as the function of EV produced by lymphoma microenvironment will be an important research path in the coming future.

\section{Genetic Alterations and MicroenVironment: the Missing LiNK}

Some of the recurrent genetic alterations in FL modulate the crosstalk between malignant $B$ cells and their supportive niche, as described above for CREBBP mutations related to the loss of MHC class II-dependent recognition by anti-tumoral $\mathrm{CD}^{\text {pos }} \mathrm{T}$ cells and for the $\mathrm{N}$-glycosylation of variable immunoglobulin genes leading to a BCR/DC-SIGN interaction bridging $B$ cells and TAM [7,49] (Figure 3). Interestingly, some somatic mutations could also functionally impact FL microenvironment and are thus related to the mechanisms of tumor niche reeducation by tumor cells. In particular loss-of-function alterations of HVEM/TNFRSF14 found in 50\% of FL patients alleviate the inhibitory signal provided by HVEM interaction with its receptor BTLA. Disruption of HVEM/BTLA interaction has been shown to trigger, in vavP-BCL2 FL mouse models and in FL patients, both B-cell autonomous activation by favoring BCR signaling but also B-cell extrinsic activation of lymphoma microenvironment [74]. In particular, HVEM inactivation triggers an amplification of BTLA ${ }^{\text {hi }}$ Tfh producing high amount of IL-4, TNF- $\alpha$ (TNF), and lymphotoxin $\alpha 1 \beta 2(L T)$. Interestingly, TNF and LT are the two non-redundant factors involved in lymphoid stroma differentiation and maintenance whereas IL-4 has been shown to induce CXCL12 upregulation in lymphoid stromal cells [58]. In agreement, FL-CAF are activated, display a lymphoid phenotype, and overexpress CXCL12. Such cell network is also reinforces by a direct impact of $B$ cells on stromal cells. In fact, whereas FL B cells could not induce CXCL12 in stromal cells, in agreement with their lack of IL-4 expression, they could contribute to their 
differentiation into lymphoid stromal cells producing CCL2 and IL-8, in a TNFdependent manner [57,61]. Interestingly, TNF production is also increased in B cells from HVEM-deficient mice. Hence, HVEM alterations contribute to the organization of a functional "ménage-à-trois" including malignant B cells, stromal cells, and Tfh. The detailed study of tumor microenvironment composition and organization in the newly developed FL/DLBCL mouse models mimicking recurrent oncogenetic hits will be highly useful to better understand the co-evolution of lymphoma cells and their permissive niche.

\section{Potential Clinical Impact of Tumor/microenvironment Crosstalk}

The identification of pro- and anti-tumoral signals delivered by the microenvironment and the understanding of how this microenvironment progressively co-evolved with tumor cells in a highly dynamic crosstalk have paved the way for the design of new therapeutic strategies. At least three main approaches with complementary impacts could be considered. The first one aims at stimulating immune effector cells. Besides pleiotropic immunomodulators, including lenalidomide, immune checkpoint inhibitors, in particular anti-PD-1/anti-PD-L1 antibodies, are under intensive evaluation in GCderived B-cell lymphomas. Interestingly, FL have been recently segregated into "inflamed" versus "uninflamed" subgroups based on mutational load and T effector gene signature, with an improved survival in the inflamed subset [75]. To what extend the identification of immunologically distinct lymphoma entities will be relevant for patient stratification in immunotherapy clinical trials deserves further investigation. More recently, new strategies emerge with the aim to stimulate TAM and/or TAN antitumor innate immune activity. As an example, mono- or bi-specific antibodies targeting SIRP- $\alpha$ and disrupting its binding to the "don't eat me" receptor CD47 expressed on lymphoma B cells are promising candidates to increase antibodydependent cell phagocytosis ([76] and unpublished data). The second strategy would be to target B-cell adhesion and/or retention within tumor niches and both the natalizumab anti-VLA-4 antibody and the disruption of the CXCL12/CXCR4 axis have shown synergistic effects with anti-CD20 antibodies $[77,78]$. Interestingly, the main $\mathrm{BCR}$ inhibitors do not only target BCR signaling but also modulate T-cell response and impair chemokine networks thus reducing B-cell retention in protective stromal microenvironment [79]. Finally, the specific targeting of microenvironment cell components, based on the better understanding of their heterogeneity and plasticity, 
is still in its infancy but has recently became a reality. Anti-TGF $\beta 1$ antibodies were recently proposed to reprogram CAF and increase T-cell infiltration within the tumor bed of urothelial tumors, thus synergizing with anti-PD-L1 antibodies [80]. In another study, chimeric antigen receptor (CAR)-T cells co-targeting tumor cells and immunosuppressive/tumor-supportive TAM, both expressing CD123 in Hodgkin's lymphomas, have paved the way for considering the use of targeted therapies redirected towards tumor microenvironment [81].

\section{CONCLUSION}

The development of high-throughput quantitative strategies, including multi-color flow cytometry, mass cytometry, functional assays on sorted cell subsets, single-cell QPCR/RNAseq strategies, or TCR sequencing, have provided new insights on the number, phenotype, and function of the main components of B-cell lymphoma microenvironment. However, new strategies are needed to elucidate the network of cell interactions that regulate in situ the organization of lymphoma cell niches, to decipher the spatial and kinetic heterogeneity of tumor microenvironment and to integrate it with corresponding tumor cell heterogeneity. In addition, the set up of relevant testing models, including lymphoma mouse models and 3D organoids mixing various cell subsets, ECM components, and mechanical constraints, is now mandatory to design adequate tailored efficient therapeutic strategies in B-NHL.

\section{Key POINTS}

- FL and DLBCL tumors are heavily infiltrated by exhausted/inhibited cytotoxic effectors and fully functional immunosuppressive cells

- FL BCR variable regions harbor mannosylated N-glycosylation sites allowing an antigen-independent engagement by microenvironment-derived lectins

- Myeloid cells, including tumor-supportive neutrophils and macrophages and myeloid-derived suppressor cells, emerge as important players in DLBCL pathogenesis

- Cancer-associated fibroblasts support directly malignant B-cell growth and organize tumor cell niche in FL patients

- Some of the recurrent genetic hits in FL favor the development of a permissive microenvironment and the crosstalk between tumor cells and their niche. 


\section{ACKNOWLEDGMENTS}

LV is a recipient of a fellowship from the Labex IGO and the Région Bretagne. KT is supported by the Fondation ARC pour la recherche sur le cancer and the French Institut National du Cancer (INCA AAP PLBIO14-40 and PLBIO17-219).

\section{REFERENCES}

1. Basso K, Dalla-Favera R. Germinal centres and B cell lymphomagenesis. Nat. Rev. Immunol. 2015, 15:172-184.

2. Scott DW, Gascoyne RD. The tumour microenvironment in B cell lymphomas. Nat. Rev. Cancer 2014, 14:517-534.

3. Tarte K. Role of the microenvironment across histological subtypes of NHL. Hematology Am Soc Hematol Educ Program 2017, 2017:610-617.

4. Amé-Thomas $\mathrm{P}$, Tarte $\mathrm{K}$. The yin and the yang of follicular lymphoma cell niches: role of microenvironment heterogeneity and plasticity. Semin. Cancer Biol. 2014, 24:23-32.

5. Huet $S$, Sujobert $P$, Salles $G$. From genetics to the clinic: a translational perspective on follicular lymphoma. Nat. Rev. Cancer 2018, 18:224-239.

6. Di Nicola M, Zappasodi R, Carlo-Stella C, et al. Vaccination with autologous tumor-loaded dendritic cells induces clinical and immunologic responses in indolent B-cell lymphoma patients with relapsed and measurable disease: a pilot study. Blood 2009, 113:18-27.

7. Green MR, Kihira S, Liu CL, et al. Mutations in early follicular lymphoma progenitors are associated with suppressed antigen presentation. Proc. Natl. Acad. Sci. U.S.A. 2015, 112:E1116-25.

** This study documents for the first time how a recurrent genetic alteration in chromatin-modifying enzymes, here inactivating mutations in CREBBP, could affect antigen presentation and T-cell activation in follicular lymphoma.

8. Challa-Malladi M, Lieu YK, Califano $\mathrm{O}$, et al. Combined genetic inactivation of $\beta 2-$ Microglobulin and CD58 reveals frequent escape from immune recognition in diffuse large B cell lymphoma. Cancer Cell 2011, 20:728-740.

9. Rimsza LM, Roberts RA, Miller TP, et al. Loss of MHC class II gene and protein expression in diffuse large B-cell lymphoma is related to decreased tumor immunosurveillance and poor patient survival regardless of other prognostic factors: a follow-up study from the Leukemia and Lymphoma Molecular Profiling Project. Blood 2004, 103:4251-4258.

10. Jiang $\mathrm{Y}$, Ortega-Molina $\mathrm{A}$, Geng $\mathrm{H}$, et al. CREBBP Inactivation Promotes the Development of HDAC3-Dependent Lymphomas. Cancer Discov 2017, 7:3853.

11. Yang Z-Z, Kim HJ, Villasboas JC, et al. Expression of LAG-3 defines exhaustion of intratumoral PD-1+ T cells and correlates with poor outcome in 
follicular lymphoma. Oncotarget 2017, 8:61425-61439.

12. Gravelle $\mathrm{P}, \mathrm{Do} \mathrm{C}$, Franchet $\mathrm{C}$, et al. Impaired functional responses in follicular lymphoma CD8(+)TIM-3(+) T lymphocytes following TCR engagement. Oncoimmunology 2016, 5:e1224044.

13. Josefsson SE, Huse $\mathrm{K}$, Kolstad $\mathrm{A}$, et al. $\mathrm{T}$ Cells Expressing Checkpoint Receptor TIGIT Are Enriched in Follicular Lymphoma Tumors and Characterized by Reversible Suppression of T-cell Receptor Signaling. Clin. Cancer Res. 2018, 24:870-881.

14. Yang Z-Z, Grote DM, Ziesmer SC, et al. IL-12 upregulates TIM-3 expression and induces $T$ cell exhaustion in patients with follicular $B$ cell non-Hodgkin lymphoma. J. Clin. Invest. 2012, 122:1271-1282.

15. Myklebust JH, Irish JM, Brody J, et al. High PD-1 expression and suppressed cytokine signaling distinguish $\mathrm{T}$ cells infiltrating follicular lymphoma tumors from peripheral T cells. Blood 2013, 121:1367-1376.

16. Kiaii S, Clear AJ, Ramsay AG, et al. Follicular lymphoma cells induce changes in T-cell gene expression and function: potential impact on survival and risk of transformation. J. Clin. Oncol. 2013, 31:2654-2661.

17. Ramsay AG, Clear AJ, Kelly G, et al. Follicular lymphoma cells induce T-cell immunologic synapse dysfunction that can be repaired with lenalidomide: implications for the tumor microenvironment and immunotherapy. Blood 2009, 114:4713-4720.

18. Yang Z-Z, Grote DM, Ziesmer SC, et al. PD-1 expression defines two distinct T-cell sub-populations in follicular lymphoma that differentially impact patient survival. Blood Cancer J 2015, 5:e281.

19. Gravelle P, Burroni B, Péricart S, et al. Mechanisms of PD-1/PD-L1 expression and prognostic relevance in non-Hodgkin lymphoma: a summary of immunohistochemical studies. Oncotarget 2017, 8:44960-44975.

20. Keane C, Gould C, Jones K, et al. The T-cell Receptor Repertoire Influences the Tumor Microenvironment and Is Associated with Survival in Aggressive Bcell Lymphoma. Clin. Cancer Res. 2017, 23:1820-1828.

* This report, based on high-throughput unbiased TCR $\beta$ sequencing, highlights for the first time a restriction of TCR repertoire associated with poor outcome in DLBCL patients.

21. Germain C, Guillaudeux T, Galsgaard ED, et al. Lectin-like transcript 1 is a marker of germinal center-derived B-cell non-Hodgkin's lymphomas dampening natural killer cell functions. Oncoimmunology 2015, 4:e1026503.

22. Amé-Thomas $\mathrm{P}$, Le Priol $\mathrm{J}$, Yssel $\mathrm{H}$, et al. Characterization of intratumoral follicular helper T cells in follicular lymphoma: role in the survival of malignant $B$ cells. Leukemia 2012, 26:1053-1063. 
23. Le K-S, Thibult M-L, Just-Landi S, et al. Follicular B Lymphomas Generate Regulatory T Cells via the ICOS/ICOSL Pathway and Are Susceptible to Treatment by Anti-ICOS/ICOSL Therapy. Cancer Res. 2016, 76:4648-4660.

24. Sage PT, Ron-Harel N, Juneja VR, et al. Suppression by TFR cells leads to durable and selective inhibition of B cell effector function. Nat. Immunol. 2016, 17:1436-1446.

25. Laurent C, Müller S, Do C, et al. Distribution, function, and prognostic value of cytotoxic T lymphocytes in follicular lymphoma: a 3-D tissue-imaging study. Blood 2011, 118:5371-5379.

26. Yang Z-Z, Novak AJ, Ziesmer SC, et al. Attenuation of CD8(+) T-cell function by $\mathrm{CD} 4(+) \mathrm{CD} 25(+)$ regulatory $\mathrm{T}$ cells in B-cell non-Hodgkin's lymphoma. Cancer Res. 2006, 66:10145-10152.

27. Yang Z-Z, Novak AJ, Ziesmer SC, et al. Malignant B cells skew the balance of regulatory T cells and TH17 cells in B-cell non-Hodgkin's lymphoma. Cancer Res. 2009, 69:5522-5530.

28. Rawal S, Chu F, Zhang M, et al. Cross talk between follicular Th cells and tumor cells in human follicular lymphoma promotes immune evasion in the tumor microenvironment. J. Immunol. 2013, 190:6681-6693.

29. Roussel $M$, Irish JM, Ménard $C$, et al. Regulatory myeloid cells: an underexplored continent in B-cell lymphomas. Cancer Immunol. Immunother. 2017, 66:1103-1111.

30. Rossille $D$, Gressier M, Damotte $D$, et al. High level of soluble programmed cell death ligand 1 in blood impacts overall survival in aggressive diffuse large BCell lymphoma: results from a French multicenter clinical trial. Leukemia 2014, 28:2367-2375.

31. Ninomiya $\mathrm{S}$, Hara $\mathrm{T}$, Tsurumi $\mathrm{H}$, et al. Indoleamine 2,3-dioxygenase in tumor tissue indicates prognosis in patients with diffuse large B-cell lymphoma treated with R-CHOP. Ann. Hematol. 2011, 90:409-416.

32. Azzaoui I, Uhel F, Rossille $\mathrm{D}$, et al. T-cell defect in diffuse large B-cell lymphomas involves expansion of myeloid-derived suppressor cells. Blood 2016, 128:1081-1092.

* This is the first phenotypic, functional, and prognostic study of circulating myeloidderived suppressor cells in DLBCL.

33. Marini $\mathrm{O}$, Spina $\mathrm{C}$, Mimiola $\mathrm{E}$, et al. Identification of granulocytic myeloidderived suppressor cells (G-MDSCs) in the peripheral blood of Hodgkin and non-Hodgkin lymphoma patients. Oncotarget 2016, 7:27676-27688.

34. Carbonnelle-Puscian A, Copie-Bergman C, Baia $\mathrm{M}$, et al. The novel immunosuppressive enzyme IL4I1 is expressed by neoplastic cells of several B-cell lymphomas and by tumor-associated macrophages. Leukemia 2009, 23:952-960. 
35. Costa A, Kieffer Y, Scholer-Dahirel A, et al. Fibroblast Heterogeneity and Immunosuppressive Environment in Human Breast Cancer. Cancer Cell 2018, 33:463-479.e10.

36. Gallouet A-S, Travert M, Bresson-Bepoldin L, et al. COX-2-independent effects of celecoxib sensitize lymphoma B cells to TRAIL-mediated apoptosis. Clin. Cancer Res. 2014, 20:2663-2673.

37. Lee MJ, Park SY, Ko JH, et al. Mesenchymal stromal cells promote B-cell lymphoma in lacrimal glands by inducing immunosuppressive microenvironment. Oncotarget 2017, 8:66281-66292.

38. Young RM, Shaffer AL, Phelan JD, et al. B-cell receptor signaling in diffuse large B-cell lymphoma. Semin. Hematol. 2015, 52:77-85.

39. Young RM, Wu T, Schmitz R, et al. Survival of human lymphoma cells requires B-cell receptor engagement by self-antigens. Proc. Natl. Acad. Sci. U.S.A. 2015, 112:13447-13454.

40. Havranek $\mathrm{O}, \mathrm{Xu} \mathrm{J}$, Köhrer $\mathrm{S}$, et al. Tonic B-cell receptor signaling in diffuse large B-cell lymphoma. Blood 2017, 130:995-1006.

41. Ruminy $P$, Etancelin $P$, Couronné $L$, et al. The isotype of the $B C R$ as a surrogate for the $\mathrm{GCB}$ and $\mathrm{ABC}$ molecular subtypes in diffuse large B-cell lymphoma. Leukemia 2011, 25:681-688.

42. Laffleur $B$, Denis-Lagache $N$, Péron $S$, et al. AID-induced remodeling of immunoglobulin genes and B cell fate. Oncotarget 2014, 5:1118-1131.

43. Dogan I, Bertocci B, Vilmont $\mathrm{V}$, et al. Multiple layers of B cell memory with different effector functions. Nat. Immunol. 2009, 10:1292-1299.

44. Pape KA, Taylor JJ, Maul RW, et al. Different B cell populations mediate early and late memory during an endogenous immune response. Science 2011, 331:1203-1207.

45. Cha S-C, Qin H, Kannan S, et al. Nonstereotyped lymphoma B cell receptors recognize vimentin as a shared autoantigen. J. Immunol. 2013, 190:48874898.

46. Coelho V, Krysov S, Ghaemmaghami AM, et al. Glycosylation of surface Ig creates a functional bridge between human follicular lymphoma and microenvironmental lectins. Proc. Natl. Acad. Sci. U.S.A. 2010, 107:1858718592.

47. Tellier J, Ménard C, Roulland S, et al. Human t(14;18)positive germinal center B cells: a new step in follicular lymphoma pathogenesis? Blood 2014, 123:3462-3465.

48. Sungalee S, Mamessier E, Morgado E, et al. Germinal center reentries of BCL2-overexpressing B cells drive follicular lymphoma progression. J. Clin. Invest. 2014, 124:5337-5351. 
49. Amin R, Mourcin F, Uhel F, et al. DC-SIGN-expressing macrophages trigger activation of mannosylated IgM B-cell receptor in follicular lymphoma. Blood 2015, 126:1911-1920.

50. Linley A, Krysov S, Ponzoni $\mathrm{M}$, et al. Lectin binding to surface Ig variable regions provides a universal persistent activating signal for follicular lymphoma cells. Blood 2015, 126:1902-1910.

51. Schneider D, Dühren-von Minden $M$, Alkhatib $A$, et al. Lectins from opportunistic bacteria interact with acquired variable-region glycans of surface immunoglobulin in follicular lymphoma. Blood 2015, 125:3287-3296.

52. Krysiak K, Gomez F, White BS, et al. Recurrent somatic mutations affecting Bcell receptor signaling pathway genes in follicular lymphoma. Blood 2017, 129:473-483.

53. Amé-Thomas $\mathrm{P}$, Hoeller $\mathrm{S}$, Artchounin $\mathrm{C}$, et al. CD10 delineates a subset of human IL-4 producing follicular helper $T$ cells involved in the survival of follicular lymphoma B cells. Blood 2015, 125:2381-2385.

54. Epron G, Amé-Thomas $\mathrm{P}$, Le Priol J, et al. Monocytes and T cells cooperate to favor normal and follicular lymphoma B-cell growth: role of IL-15 and CD40L signaling. Leukemia 2012, 26:139-148.

55. Manfroi B, McKee T, Mayol JF, et al. CXCL-8/IL8 Produced by Diffuse Large Bcell Lymphomas Recruits Neutrophils Expressing a Proliferation-Inducing Ligand APRIL. Cancer Res. 2017, 77:1097-1107.

56. Hirz T, Matera E-L, Chettab K, et al. Neutrophils protect lymphoma cells against cytotoxic and targeted therapies through CD11b/ICAM-1 binding. Oncotarget 2017, 8:72818-72834.

57. Grégoire $\mathrm{M}$, Guilloton $\mathrm{F}$, Pangault $\mathrm{C}$, et al. Neutrophils trigger a NF-KB dependent polarization of tumor-supportive stromal cells in germinal center Bcell lymphomas. Oncotarget 2015, 6:16471-16487.

58. Pandey S, Mourcin F, Marchand T, et al. IL-4/CXCL12 loop is a key regulator of lymphoid stroma function in follicular lymphoma. Blood 2017, 129:25072518.

* This is the first report of phenotypic and functional modifications of FL-infiltrating stromal cells in invaded lymph nodes and bone marrow. The upregulation of CXCL12 in FL-CAF is associated with malignant B-cell recruitment, adhesion, and activation.

59. Mourcin F, Pangault C, Amin-Ali R, et al. Stromal cell contribution to human follicular lymphoma pathogenesis. Front Immunol 2012, 3:280.

60. Tian YF, Ahn H, Schneider RS, et al. Integrin-specific hydrogels as adaptable tumor organoids for malignant B and T cells. Biomaterials 2015, 73:110-119.

** This study provides an elegant 3D lymphoma organoid model integrating lymphoid-like stromal cells and adhesive peptides with distinct integrin specificities. 
This adaptable system reveals that $B$ and $T$ cell lymphomas have different prosurvival integrin signaling requirements and would be useful to screen new drugs.

61. Guilloton F, Caron G, Ménard C, et al. Mesenchymal stromal cells orchestrate follicular lymphoma cell niche through the CCL2-dependent recruitment and polarization of monocytes. Blood 2012, 119:2556-2567.

62. Brady MT, Hilchey SP, Hyrien O, et al. Mesenchymal stromal cells support the viability and differentiation of follicular lymphoma-infiltrating follicular helper $\mathrm{T}$ cells. PLOS ONE 2014, 9:e97597.

63. Wang $X$, Rodda LB, Bannard $\mathrm{O}$, et al. Integrin-mediated interactions between $B$ cells and follicular dendritic cells influence germinal center B cell fitness. J. Immunol. 2014, 192:4601-4609.

64. Sangaletti $S$, Tripodo $C$, Portararo $P$, et al. Stromal niche communalities underscore the contribution of the matricellular protein SPARC to B-cell development and lymphoid malignancies. Oncoimmunology 2014, 3:e28989.

65. Sangaletti S, Tripodo C, Vitali C, et al. Defective stromal remodeling and neutrophil extracellular traps in lymphoid tissues favor the transition from autoimmunity to lymphoma. Cancer Discov 2014, 4:110-129.

* This study demonstrates that faulty extracellular matrix associated with SPARC downregulation can facilitate lymphoid malignancies in mice predisposed to autoimmunity and could contribute to lymphoma development in human.

66. Lenz G, Wright G, Dave SS, et al. Stromal gene signatures in large-B-cell lymphomas. N. Engl. J. Med. 2008, 359:2313-2323.

67. Weissmann M, Arvatz G, Horowitz N, et al. Heparanase-neutralizing antibodies attenuate lymphoma tumor growth and metastasis. Proc. Natl. Acad. Sci. U.S.A. 2016, 113:704-709.

68. Gravelle $\mathrm{P}$, Jean $\mathrm{C}$, Familiades J, et al. Cell growth in aggregates determines gene expression, proliferation, survival, chemoresistance, and sensitivity to immune effectors in follicular lymphoma. Am. J. Pathol. 2014, 184:282-295.

69. Astarita JL, Cremasco V, Fu J, et al. The CLEC-2-podoplanin axis controls the contractility of fibroblastic reticular cells and lymph node microarchitecture. Nat. Immunol. 2015, 16:75-84.

70. Salmon H, Franciszkiewicz K, Damotte D, et al. Matrix architecture defines the preferential localization and migration of $\mathrm{T}$ cells into the stroma of human lung tumors. J. Clin. Invest. 2012, 122:899-910.

71. Paggetti J, Haderk F, Seiffert $M, J$ et al. Exosomes released by chronic lymphocytic leukemia cells induce the transition of stromal cells into cancerassociated fibroblasts. Blood 2015, 126:1106-1117.

72. Aung $\mathrm{T}$, Chapuy $\mathrm{B}$, Vogel $\mathrm{D}$, et al. Exosomal evasion of humoral immunotherapy in aggressive B-cell lymphoma modulated by ATP-binding 
cassette transporter A3. Proc. Natl. Acad. Sci. U.S.A. 2011, 108:15336-15341.

73. Manček-Keber M, Lainšček D, Benčina M, et al. Extracellular vesicle-mediated transfer of constitutively active MyD88L265P engages MyD88wt and activates signaling. Blood 2018, 131:1720-1729.

74. Boice M, Salloum D, Mourcin F, et al. Loss of the HVEM Tumor Suppressor in Lymphoma and Restoration by Modified CAR-T Cells. Cell 2016, 167:405418.e13.

** This study demonstrates that HVEM mutations found in $40 \%$ of FL patients favor lymphomagenesis through both direct B-cell activation and indirect stimulation of a permissive microenvironment including Tfh and stromal cells. It also proposes that CAR-T cells engineered to produce solHVEM are active against lymphoma.

75. Bolen CR, McCord R, Huet S, et al. Mutation load and an effector T-cell gene signature may distinguish immunologically distinct and clinically relevant lymphoma subsets. Blood Adv 2017, 1:1884-1890.

76. Ring NG, Herndler-Brandstetter $\mathrm{D}$, Weiskopf $\mathrm{K}$, et al. Anti-SIRPa antibody immunotherapy enhances neutrophil and macrophage antitumor activity. Proc. Natl. Acad. Sci. U.S.A. 2017, 114:E10578-E10585.

77. Mraz $\mathrm{M}$, Zent $\mathrm{CS}$, Church $\mathrm{AK}$, et al. Bone marrow stromal cells protect lymphoma B-cells from rituximab-induced apoptosis and targeting integrin a-4$\beta-1$ (VLA-4) with natalizumab can overcome this resistance. Br. J. Haematol. 2011, 155:53-64.

78. O'Callaghan $\mathrm{K}$, Lee $\mathrm{L}$, Nguyen $\mathrm{N}$, et al. Targeting CXCR4 with cell-penetrating pepducins in lymphoma and lymphocytic leukemia. Blood 2012, 119:17171725.

79. Maharaj K, Sahakian E, Pinilla-lbarz J: Emerging role of BCR signaling inhibitors in immunomodulation of chronic lymphocytic leukemia. Blood Adv 2017, 1:1867-1875.

80. Mariathasan S, Turley SJ, Nickles D, et al. TGF $\beta$ attenuates tumour response to PD-L1 blockade by contributing to exclusion of T cells. Nature 2018, 554:544-548.

81. Ruella $\mathrm{M}$, Klichinsky $\mathrm{M}$, Kenderian SS, et al. Overcoming the Immunosuppressive Tumor Microenvironment of Hodgkin Lymphoma Using Chimeric Antigen Receptor T Cells. Cancer Discov 2017, 7:1154-1167. 


\section{FiguRES TITLES AND LEGENDS}

Figure 1. Interactions of malignant $B$ cells with immune microenvironment

B-NHL niches exhibit features of both defective anti-tumoral and activated protumoral microenvironment. First, infiltrating immune effectors are characterized by upregulation of co-inhibitory receptors, impairment of actin polymerization at the immunological synapse, impaired motility, and decreased TCR diversity. In addition, FL and DLBCL tumors are heavily infiltrated by immune suppressive cells including Treg/Tfr and heterogeneous myeloid suppressive cells, which could also be detected in peripheral blood as myeloid derived suppressor cells (MDSC). Importantly, tumorassociated macrophages (TAM) and neutrophils (TAN) also directly contribute to malignant B cell growth and drug resistance through the production of cytokines, adhesion molecules, and lectins. Similarly, Tfh are a major FL B-cell supportive cell compartment and contribute directly and indirectly to lymphomagenesis through the activation of $B$ cells, stromal cells, and TAM.

Figure 2. Cancer-associated fibroblasts play a key role in tumor permissive microenvironment

Cancer-associated fibroblasts (CAF) could directly favor malignant B cell homing and survival through the production of chemokines, Hh ligands, BAFF, and adhesion molecules. In addition they should be considered as organizers of lymphoma cell niche by triggering recruitment, and differentiation/polarization of Tfh, TAM, and TAN, whereas they could contribute to the inhibition of immune effector cell activity. Conversely, malignant $\mathrm{B}$ cells themselves but also other components of the tumor niche contribute to the activation of CAF. Importantly, besides stromal cells, acellular components, including extracellular matrix (ECM) and extracellular vesicles, participate to the construction and architecture of lymphoma cell niche.

Figure 3. Genetic alterations subvert tumor microenvironment in follicular lymphoma

At least three recurrent genetic hits modulate the crosstalk between malignant $B$ cells and their supportive niche in follicular lymphoma (FL) including: i) mutations of CREBBP associated with a loss of MHC class II expression; ii) introduction of $\mathrm{N}$ glycosylation sites in the variable region of $F L B C R$ that are occupied by 
oligomannoses and allow antigen-independent/BCR-dependent interactions with DCSIGN ${ }^{\text {pos }}$ tumor associated macrophages (TAM); and iii) HVEM inactivation increasing BCR signaling and triggering amplification of IL-4 ${ }^{\text {hi }} \mathrm{TNF}^{\mathrm{hi}} \mathrm{LT}^{\mathrm{hi}}$ Tfh which, in turn, activate tumor $B$ cells and CAF. 


\section{Figure 1}

\section{Activated Pro-Tumoral Microenvironment}

Inhibition of anti-tumor immunity Inhibition of Tfh

Production of B-cell supportive molecules Activation of TAM and stromal cells
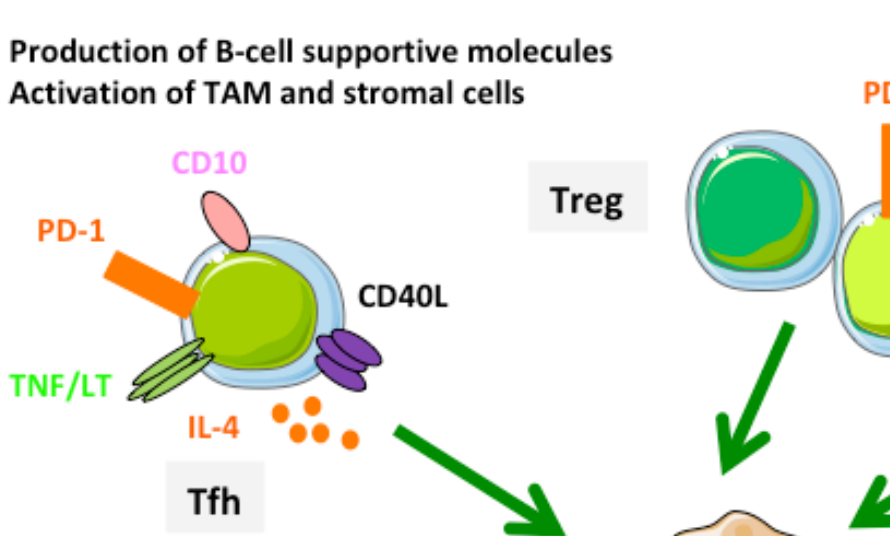

Treg

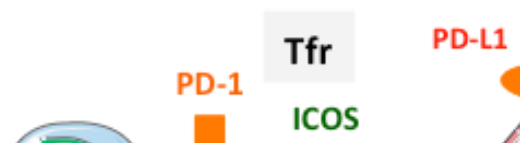




\section{Figure 2}

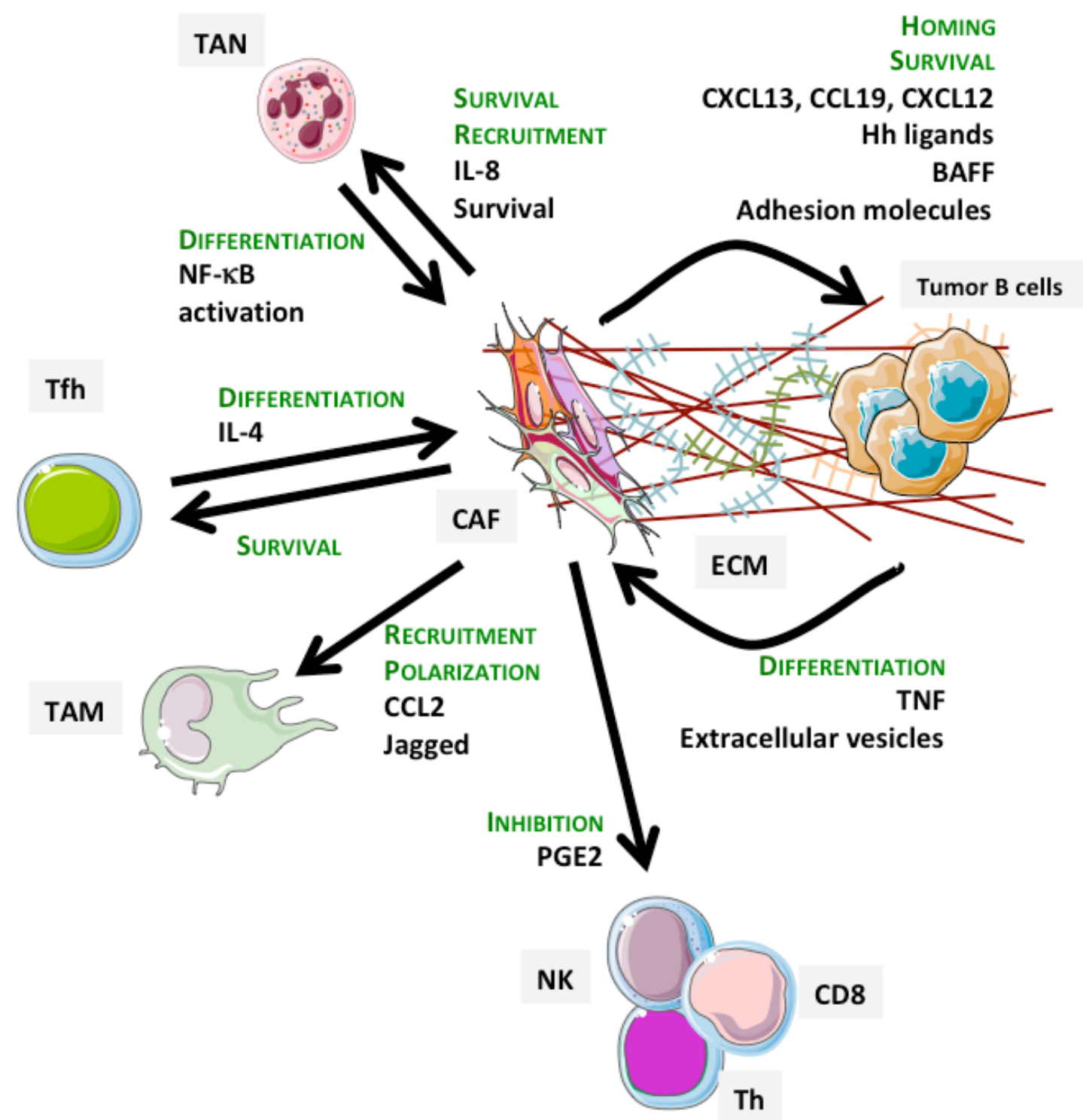




\section{Figure 3}

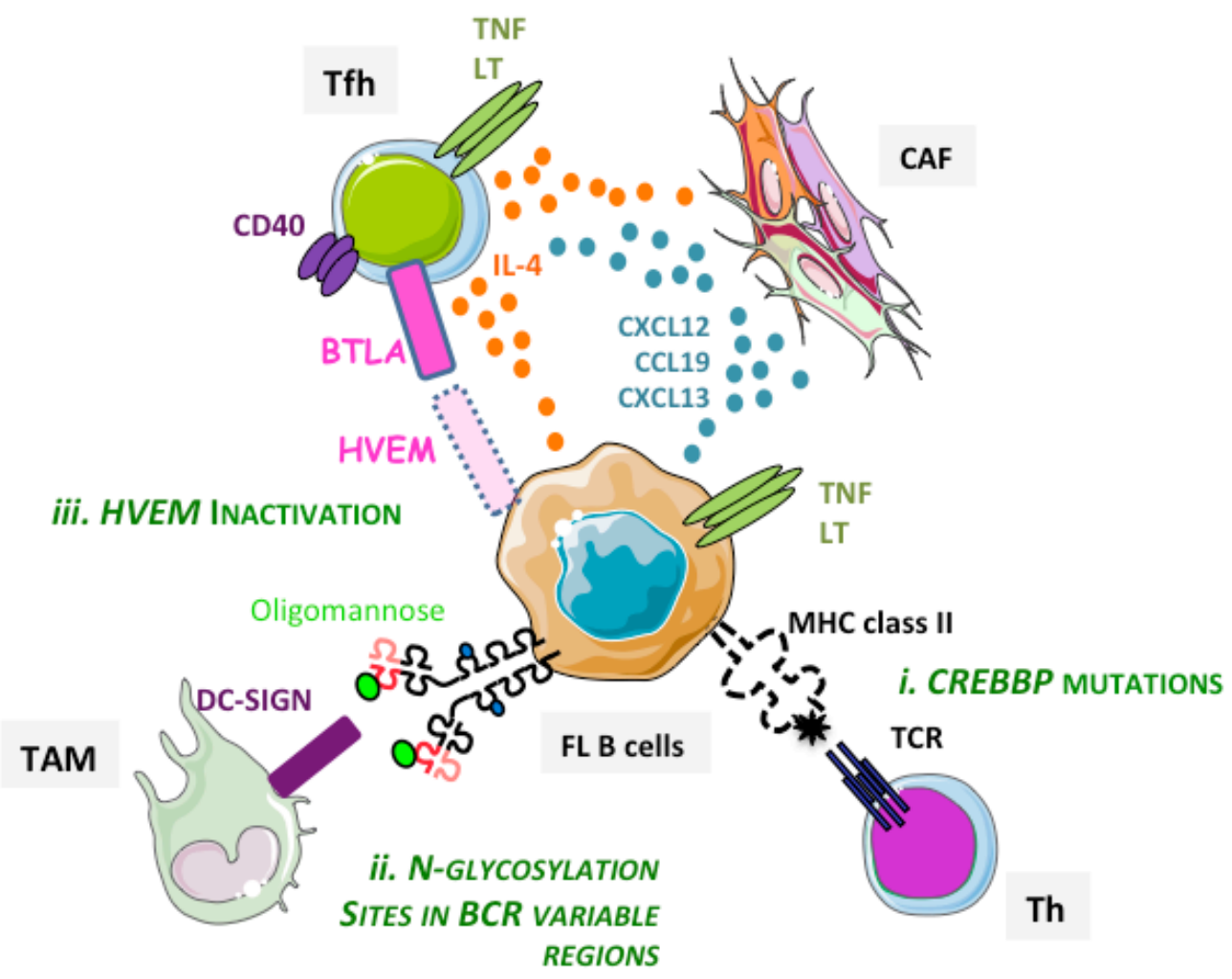

